\title{
Downregulation of microRNA-155 by preoperative administration of valproic acid prevents postoperative seizures by upregulating SCN1A
}

\author{
ZHIJIE ZHANG ${ }^{1}$, ZHENZHONG WANG $^{1}$, BO ZHANG $^{1}$ and YAN LIU ${ }^{2}$ \\ ${ }^{1}$ Department of Neurosurgery, The Affiliated Yangming Hospital of Ningbo University; \\ ${ }^{2}$ Department of Neurosurgery, Ningbo Yinzhou District No. 2 Hospital, Ningbo, Zhejiang 315100, P.R. China
}

Received April 5, 2016; Accepted September 20, 2017

DOI: $10.3892 / \mathrm{mmr} .2017 .8004$

\begin{abstract}
The risk of seizure is increased following brain surgery such as cranioplasty. Patients with seizures that are treated with valproic acid (VPA) may have a decreased risk of further seizures. To verify microRNA (miR)-155 as a potential biomarker for the occurrence of seizures, reverse transcription quantitative polymerase chain reaction (RT-qPCR) was used. Computational analysis and luciferase reporter assay was performed to identify the putative target of miR-155. RT-qPCR and western blot analyses were used to determine the expression level of miR-155, sodium voltage-gated channel $\alpha$ subunit 1 (SCN1A) mRNA and protein. RT-qPCR analysis indicated that miR-155 levels in patients who experienced seizures increased 2.45-fold compared with patient who did not experience seizures, indicating miR-155 may be a potential biomarker for the occurrence of seizures. SCN1A was identified as a target gene of miR-155; the luciferase reporter assay revealed a negative regulatory relationship between miR-155 and SCN1A. The expression of SCN1A mRNA of patients receiving VPA was higher compared with the control group patients. Furthermore, the expression levels of SCN1A mRNA and protein were reduced or elevated following transfection with miR-155 mimics or inhibitors, respectively, compared with the scramble control. Furthermore, a concentration-dependent effect of miR-155 on the expression of SCN1A was observed. In conclusion, miR-155 may be associated with the risk of seizure and SCN1A may be a target gene of miR-155. Downregulation of microRNA-155 by preoperative administration of VPA may prevent postoperative seizure by upregulating the expression of SCN1A.
\end{abstract}

Correspondence to: Dr Yan Liu, Department of Neurosurgery, Ningbo Yinzhou District No. 2 Hospital, 2 Qianhe Road, Ningbo, Zhejiang 315100, P.R. China

E-mail: 925403713@qq.com

Key words: microRNA-155, valproic acid, sodium voltage-gated channel $\alpha$ subunit 1

\section{Introduction}

Cranioplasty is the predominant method for the treatment of a variety of skull defects. Seizure is a common complication of skull injury and cranioplasty, with the incidence ranging between 14.8 and $33.0 \%(1,2)$. Seizures pose a serious threat to human health, with patients subsequently presenting with varying degrees of mental disorders and physical damage, which greatly impacts upon their normal learning and quality of life, and may bring serious mental and economic burdens to society and the patient's family. Seizures are a frequent neurological problem during the neonatal period, occurring in 1.8-5 out of 1,000 live births in Canada and The United States (3). The most common cause of neonatal seizures is hypoxic/ischemic encephalopathy, affecting $1-2$ out of 1,000 live neonatal births, therefore contributing to $\sim 2 / 3$ of seizure cases in neonatal babies (3). Clinically, it can be challenging to diagnose neonatal seizures, which may be exclusively electrographic (4). In addition, currently available antiepileptic drugs (AEDs) are often not effective for neonatal seizures (5). The frequency of seizure has been demonstrated to be significantly elevated following cranioplasty, and previous research has indicated that valproic acid could decrease the risk of post-operative seizures (6).

Recently, microRNAs (miRNAs) have been identified as pivotal regulators of gene function, and this has been a significant step forward in our understanding of gene regulatory mechanisms $(7,8)$. Although there are only a few hundred known miRNAs, it is estimated that miRNAs may regulate $>1 / 3$ of all human genes, as each miRNA is likely to regulate hundreds of target genes $(9,10)$. miRNAs are involved in several intrinsic cellular processes, including cellular metabolism, immune responses, hematopoietic differentiation and cell-cycle regulation (11-14), and their expression patterns have been investigated in schizophrenia, Down's syndrome and a variety of tumors (15-17). Previous research demonstrated that valproic acid (VPA) was able to modulate miRNA expression profiles in rats (18). The interaction between miRNAs and their target genes is closely modulated, and our recent report investigated this using in silico methods to predict the network of miRNAs that interacted with and interrupted protein complexes following VPA administration (19). Furthermore, a 
previous study revealed that VPA was able to affect expression of miRNAs in the brain (18).

Based on these observations, the present study compared patients who underwent cranioplasty from March 2010 to March 2015 and used AEDs to control preoperative or postoperative cranioplasty-related seizures, with patients who did not use AED drugs across the same period. The role of miR155 and its potential target SCN1A in the control of post-operative seizure were explored. Downregulation of microRNA-155 by preoperative administration of VPA prevents postoperative seizure by upregulating SCN1A.

\section{Materials and methods}

Subjects and inclusion/exclusion criteria. All patients who came to the Department of Neurosurgery, the Affiliated Yangming Hospital of Ningbo University (Ningbo, China) for cranioplasty from March 2010 to March 2015, and were self reliant in their ability to take care of themselves and to perform basic self-care were suitable for inclusion in the present study. A total of 85 cases were recruited into the study; 2 patients were lost to follow-up and 3 were excluded due to rejection reaction and infection, leaving 80 cases [57 males, 23 females; age 36.2 \pm 2.6 (range 17-72) years]. A total of 69 cases had subdural (or intracerebral) hematoma clearance + decompressive craniectomy due to brain trauma, and 11 had intracranial hematoma clearance + decompressive craniectomy due to intracranial spontaneous bleeding. Patients underwent craniotomies in the previous 3-25 months (average, $4.4 \pm 0.7$ months) prior to undergoing the present cranioplasty. The patients presented with several skull defect sites: 52 cases of unilateral frontotemporal and parietal, 15 cases of bilateral frontotemporal and parietal, 9 cases of bilateral frontal, 3 cases of unilateral occipital and 1 case of bilateral occipital. Amongst all subjects, tissue samples were available for 48 participants (VPA treated, $n=23$; Control, $n=25$ ). The study protocol was approved by the research ethics committee at The Affiliated Yangming Hospital of Ningbo University (Ningbo, China), and written informed consent was obtained from each participant.

Patients who presented with the following were excluded: Patients who had hydrocephalus shunt under anesthesia; patients who had seizures prior to being hospitalized for the present cranioplasty, including those who had or had not used drug control; patients who had their implant titanium plate and nail for cranioplasty removed due to infection or rejection reaction following cranioplasty; and patients who had severe heart, liver or kidney dysfunctions or had routine blood abnormalities prior to surgery.

All enrolled patients were randomly divided into experimental and control groups. A few patients were not included in the statistics: 2 cases in the control group were lost to follow-up and 2 cases underwent reoperation due to postoperative rejection; 1 case in the experimental group developed an infection. There were 41 cases remaining in the VPA-treated group with 30 male cases and 11 female cases. SCN1A expression was detected in 23 of them. Patients received craniotomies in the previous 3-20 months (average, 4.2 \pm 0.5 months) prior to undergoing the present cranioplasty. The experimental patients presented with the following skull defects: 26 cases of unilateral frontotemporal and parietal, 9 cases of bilateral frontotemporal and parietal, 4 cases of bilateral frontal, 2 cases of other sites. The defect area of each bone window was between $4 \times 4$ and $12 \times 16 \mathrm{~cm}$ (average, $9.5 \times 10.2 \mathrm{~cm}$ ). There were 39 cases remaining in the control group, with 28 male and 11 female cases. SCN1A expression was detected in 25 of them. Patients received craniotomies in the previous 3.3-25 months (average, $4.6 \pm 0.8$ months) prior to undergoing the present cranioplasty. The control patients presented with the following skull defects: 26 cases of unilateral frontotemporal and parietal, 6 cases of bilateral frontotemporal and parietal, 5 cases of bilateral frontal, 2 cases of other sites. The defect area of each bone window was between $4 \times 5$ and $13 \times 15 \mathrm{~cm}$ (average, $9.4 \times 10.5 \mathrm{~cm}$ ). The two groups of patients had no significant difference in clinical features (data not shown).

Surgical approach. Patients underwent clinical examination following admission, including liver and kidney function analyses, routine blood tests and head computed tomography scan. Patients were fitted for custom titanium mesh plates, and underwent operation $\sim 5$ days later. Under general anesthesia, the scalp was cut open along the original surgical incision line to the skull, and the scalp and temporal muscle were carefully peeled to expose the endocranium and fully expose the bone window surface. The custom titanium (titanium mesh) plate was placed on the skull window and was fixed with a few titanium nails. Outside the plate a drainage tube was connected with negative pressure (to be removed $48 \mathrm{~h}$ later). All patients were started on medicine 4 days prior to surgery (following admission and whilst the custom titanium mesh plate was prepared) until 1 month following surgery. The control group took oral $20 \mathrm{mg}$ ATP tablet (tid). The experimental group took ATP (20 mg) and Compound Sodium Valproate and Valproic Acid Sustained Release tablets [500 mg (bid); Sanofi-Aventis (Hangzhou) Pharmaceuticals Co., Ltd., Hangzhou, China].

Efficacy evaluation. The following clinical parameters were observed and compared between the two groups of patients: Incidence of seizures following admission (4 days prior to surgery) up to 1 month following surgery; abnormal rate of liver function; routine blood tests or other indicators 2 weeks post-surgery (serum aspartate aminotransferase $>2$-fold of the preoperative level was considered abnormal; white blood cell or platelet count reduced by $\geq 50 \%$ of the preoperative level was considered abnormal).

Cell culture and transfection. U251 cells (American Type Culture Collection, Manassas, VA, USA) were selected for the analysis due to low levels of expression of miR-155. U251 cells were grown in Dulbecco's modified Eagle's medium (Invitrogen; Thermo Fisher Scientific, Inc., Waltham, MA, USA), supplemented with $1 \%$ penicillin/streptomycin, $2 \mathrm{mM}$ glutamine and $10 \%$ fetal bovine serum (Invitrogen; Thermo Fisher Scientific, Inc.) at $37^{\circ} \mathrm{C}$ with $5 \% \mathrm{CO}_{2} / 95 \%$ air. When confluence reached 80\%, Lipofectamine 2000 (Invitrogen; Thermo Fisher Scientific, Inc.) was used to transfect U251 cells with miRNA (miR)-155 mimics (5'UUAAUGCUA AUCGUGAUAGGGG3'), negative control (NC; 5'CAGUAC UUUUGUGUAGUACAA3') and miR-155 inhibitors (5'CCC CUAUCACGAUUAGCAUUAA3'), in accordance with the 
manufacturer's protocol (GenePharma, Shanghai, China). The final concentration of miR-155, NC and inhibitor is $100 \mathrm{nM}$. Three independent experiments were performed.

$R N A$ isolation and reverse transcription quantitative polymerase chain reaction ( $R T-q P C R)$. TRIzol reagent (Invitrogen; Thermo Fisher Scientific, Inc.) was used to purify total RNA from U251 cells $\left(5 \times 10^{6}\right.$ cells) and cerebrospinal fluid (CSF) samples $(2 \mathrm{ml})$ in accordance with the manufacturer's protocol. CSF was sampled via epidural during surgery. All CSF samples were put on ice immediately and spun within $15 \mathrm{~min}$ at $500 \mathrm{x} \mathrm{g}$ for $10 \mathrm{~min}$; the supernatant was stored at $-80^{\circ} \mathrm{C}$ until further use. DNaseI treatment (Takara Bio Inc., Otsu, Japan) was used to treat the total RNA, according to the manufacturer's protocol. A NanoDrop (Thermo Fisher Scientific, Inc., Wilmington, DE, USA) was used to assess the concentration and quality of RNA. QuantiTect_SYBR_Green RT-PCR kit (Qiagen GmbH, Hilden, Germany) was used to synthesize cDNA from 200 ng total RNA, in accordance with the manufacturer's protocol. An Applied Biosystems 7500 Sequence Detection System (Thermo Fisher Scientific, Inc., USA) was used to perform qPCR. The upstream primers (5'-3') used in the study were: TTAATGCTAATCGTGATAGG for miR-155; AATTGCACTCGTCCCGGCCTCC for miR-92b; CCTCTGGGCCCTTCCTCCAG for miR-326; CTGGCC CTCTCTGCCCTTCCGT for miR-328; TATGACTGATGT GTGCGTGTGTCTG for miR-468; AGGGATCGCGGGCGG GTGGCGGCCT for miR-638; AGGCGGGGCGCCGCG GGACCGC for miR-663; GTGCGGAACGCTGGCCGG GGCG for miR-685. Downstream primer was the universal primer supplied by Qiagen $\mathrm{GmbH}$. The thermocycling reaction involved $95^{\circ} \mathrm{C}$ for $10 \mathrm{~min}$ (initial denaturation), followed by 40 cycles of $95^{\circ} \mathrm{C}$ for $15 \mathrm{sec}$ and $60^{\circ} \mathrm{C}$ for $60 \mathrm{sec}$. Small nuclear RNA U6 (5'CTCGCTTCGGCAGCACA3' and 5'AAC GCTTCACGAATTTGCGT3') was used as an internal control to normalize the expression of microRNAs.

For SCN1A detection, each sample was reverse transcribed into cDNA and analyzed by SYBR-Green Real-Time PCR kit (Bio-Rad, America). Quantitative real-time PCR was performed using Applied Biosystems 1900 system (Thermo Fisher Scientific, Inc). The primers for SCN1A were 5'GCA TCCGTGGCTCCCTATTTT3' and 5'CTCATTGCTCGT TGCCTTTGG3'. GAPDH (5'CCACTCCTCCACCTTTGAC 3 and 5'ACCCTGTTGCTGTAGCCA3') was used as an internal control for-SCN1A. The $2^{-\Delta \Delta C q}$ method (20) was used to analyze the relative quantification of miR-155 and SCN1A mRNA. All reactions were performed in triplicate.

Luciferase assay. The University of California Santa Cruz genome browser (http://genome.ucsc.edu) was used to examine the downstream region of miR-155. The predicted miR-155 binding site in the 3'-untranslated region (UTR) of SCN1A was amplified using PCR (5'CGCTCGAGATGAAAATAA ATAAAAATAATTGG3' and 5'ATAAGCTTGCTAAAA TAAAAAATGTAAT3', underlined are $\mathrm{XhoI}$ and HindIII sites respectively, $2103 \mathrm{bp}$ ). The reaction condition was $95^{\circ} \mathrm{C}$ $2 \mathrm{~min}$, followed by 30 cycles of $95^{\circ} \mathrm{C} 30 \mathrm{sec}, 53^{\circ} \mathrm{C} 30 \mathrm{sec}$ and $72^{\circ} \mathrm{C} 2 \mathrm{~min} .3^{\prime} \mathrm{UTR}$ mutants were created using a site directed mutagenesis kit (Takara Bio Inc.) (Fig. 1). The PCR products and the site-directed mutagenesis products were inserted

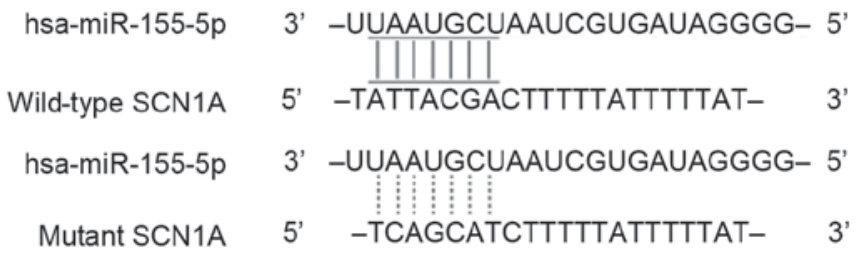

Figure 1. SCN1A contains a putative seed sequence for miR-155 in the 3'untranslated region. Hsa, Homo sapiens; miR, microRNA; SCN1A, sodium voltage-gated channel $\alpha$ subunit 1 .

into the XhoI/HindIII restriction sites of a pGL3-Basic vector (Promega Corporation, Madison, WI, USA) to create the wild-type or mutant luciferase/reporter constructs. Sequencing was performed to confirm all the constructs. Lipofectamine 2000 (Invitrogen; Thermo Fisher Scientific, Inc.) was used to co-transfect the U251 cells with wild-type SCN1A 3'UTR or mutant SCN1A 3'UTR luciferase reporter constructs at a final concentration of $100 \mathrm{nM}$ per well, together with miR-155 mimic or miR-NC with a final concentration of $100 \mathrm{nM}$. The Dual-Luciferase Reporter Assay System (Promega Corporation) was used to measure the activities of firefly and Renilla luciferase $48 \mathrm{~h}$ following transfection. Three independent experiments were performed.

Western blot analysis. The expression of SCN1A protein was assessed using western blot analysis. PBS was used to wash the U251 cells and tissue samples twice, and U251 cells $\left(5 \times 10^{6}\right.$ cells) were lysed using radioimmunoprecipitation assay lysis buffer (Sigma-Aldrich; Merck KGaA) containing Halt Protease Inhibitor Cocktail (Thermo Fisher Scientific, Inc.) according to the manufacturer's protocol. Cellular lysates were centrifuged for $15 \mathrm{~min}$ at $1,500 \mathrm{xg}$ at $4^{\circ} \mathrm{C}$ to obtain the supernatants. A DC Protein assay (Bio-Rad Laboratories, Inc., Hercules, CA, USA) was used to determine the concentration of protein. Proteins were heat-denatured in boiling water. Proteins ( $50 \mu \mathrm{g} / \mathrm{lane})$ were separated by $10 \%$ SDS-PAGE and transferred to an Immobilon-P membrane (EMD Millipore, Billerica, MA, USA) according to the manufacturer's protocol. PBS containing 5\% skimmed milk and $0.1 \%$ Tween-20 was used to block the membrane to avoid non-specific binding. Membranes were incubated with primary rabbit monoclonal antibodies against SCN1A (cat. no. 14380; 1:5,000) and $\beta$-actin (cat. no. 4970; 1:15,000), both from Cell Signaling Technology (Danvers, MA, USA), at $4^{\circ} \mathrm{C}$ for $12 \mathrm{~h}$. PBS containing $0.1 \%$ Tween-20 was subsequently used to wash the membranes three times for 10 min each and the membranes were incubated with the secondary antibody (cat. no. 14708; 1:8,000; Cell Signaling Technology, Danvers, MA, USA) at $37^{\circ} \mathrm{C}$ for $2 \mathrm{~h}$. The blots were subsequently washed with PBS containing 0.1\% Tween-20. Enhanced Chemiluminescence Detection Reagent (GE Healthcare Life Sciences, Little Chalfont, UK) was used to detect the bound antibody in accordance with the manufacturer's protocol.

Statistical analysis. Data are presented as the mean \pm standard deviation. Statistical significance was tested using either one-way analysis of variance followed by a Bonferroni post-hoc test, chi-square statistic or paired Student's t-test 
Table I. Comparisons of clinical data of experimental and control groups.

\begin{tabular}{lcccc}
\hline Clinical data & Experimental group $(\mathrm{n}=41)$ & Control group $(\mathrm{n}=39)$ & $\chi^{2}$ & P-value \\
\hline Seizure, $\mathrm{n}(\%)$ & $3(7.3)$ & $11(28.2)$ & 6.04 & 0.019 \\
Abnormal liver function, $\mathrm{n}(\%)$ & $3(7.3)$ & $2(5.1)$ & 1.61 & 1.00 \\
Abnormal routine blood, $\mathrm{n}(\%)$ & $1(2.4)$ & $1(2.6)$ & 0.01 & 1.00 \\
\hline
\end{tabular}

A

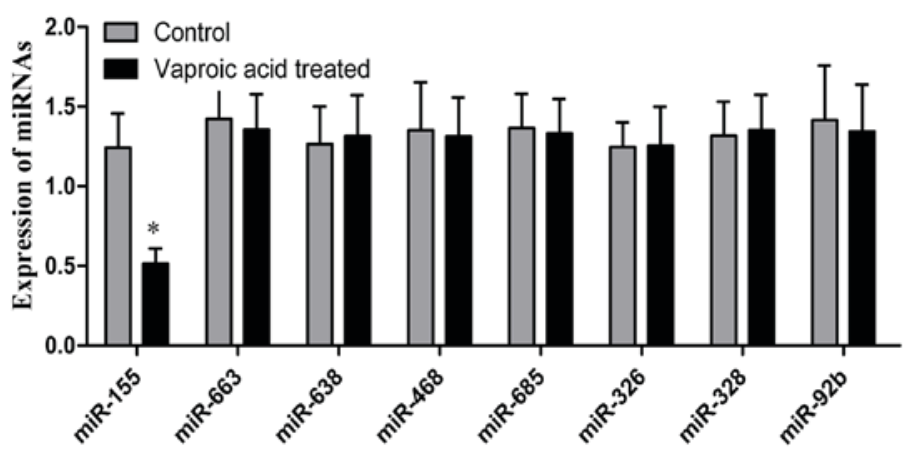

B

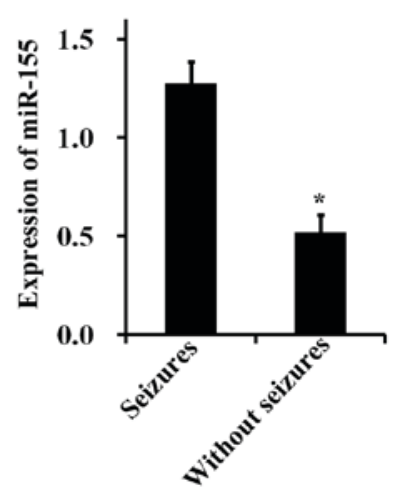

Figure 2. Expression of miR-155 was assayed by RT-qPCR. (A) miR-155 exhibits markedly different expression in patients in the untreated control and valproic acid-treatment groups. $\mathrm{P}<0.05$ vs. the control group. (B) miR-155 exhibits markedly different expression in patients who experienced seizures and patients who did not experience seizures. miR, microRNA. ${ }^{*} \mathrm{P}<0.05$ vs. patients with seizures.

(two-tailed). Relationships between two variables from the same test were evaluated by the linear Pearson correlation coefficient (r) and multiple regression analyses. JMP Pro 12 (SAS Institute, Cary, NC, USA) was used to analyze the data. $\mathrm{P}<0.05$ was considered to indicate a significant difference.

\section{Results}

Evaluation of the preventive effects of VPA on post-operative seizure. There were 11 cases of seizures in the control group, with an incidence of $28.2 \%$ (Table I), including 4 cases of preoperative seizures and 7 cases within 1 month of surgery ( 6 cases within 2 weeks of surgery; one case 2 weeks post-surgery). There were 3 cases of seizures in the experimental group, with an incidence of $\sim 7.3 \%$, including one case of preoperative seizure and two cases within 1 month of surgery (one case within 2 weeks of surgery, one case 2 weeks post-surgery). The difference in the incidence rates of seizures between the groups was statistically significant $(\mathrm{P}=0.019$; Table I). Following surgery, there were 2 cases of abnormal liver function in the control group, and 3 cases in the experimental group. There was one case of abnormal routine blood analysis (reduced white blood cells) in each of the two groups. There were no significant differences identified for liver function or routine blood analysis between the two groups (Table I).

Deregulation of miR-155 in seizure. RT-qPCR was used to investigate differentially expressed miRNAs in subjects of control and VPA-treated patients. A total of 8 miRNAs (miRNA-155, miRNA-633, miRNA-638, miRNA-468, miRNA-685, miRNA-326, miRNA-328 and miRNA-92b) closely associated with seizure were analyzed by RT-qPCR (Fig. 2). miR-155 was the only significantly different miRNA between control and VPA-treated groups and the expression of miR-55 decreased 2.5 fold in the VPA-treated group when compared with it in the control group. The remaining 7 miRNAs demonstrated similar expression levels between the two groups. These results suggested that miR-155 may be a potential biomarker for seizure development.

miR-155 downregulation may be associated with seizure occurrence. The expression of miR-155 in patients who experienced seizures was 2.45 folds higher compared with patients who did not experience seizures among all patients (Fig. 2B). Pearson correlation analysis among all patients revealed a positive association between miR-155 expression and seizure occurrence $(r=0.503, \mathrm{P}=0.018)$. Furthermore, a multivariate linear model indicated the relationship between miR-155 and seizure was persistent in the control $(\mathrm{r}=0.490, \mathrm{P}=0.029)$ and experimental groups $(\mathrm{r}=0.513, \mathrm{P}=0.014)$, indicating that increased miR-155 expression may be associated with seizure occurrence.

SCN1A may be a target of miR-155. Online miRNA target prediction tools were used to search for target genes of miR-155, and SCN1A was identified as a candidate target gene of miR-155, as it contained the appropriate seed sequence in the 3'UTR (Fig. 1). In addition, it was confirmed that the miR-155 mimics or miR-NC were successfully transfected into U251 cells (Fig. 3A). A luciferase reporter assay was used to investigate the regulatory relationship between miR-155 and SCN1A. The luciferase activity of cells transfected with wild-type SCN1A 3'UTR and miR-155 mimics 
A

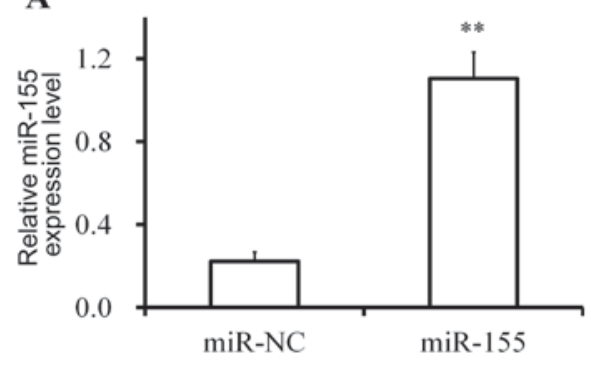

B

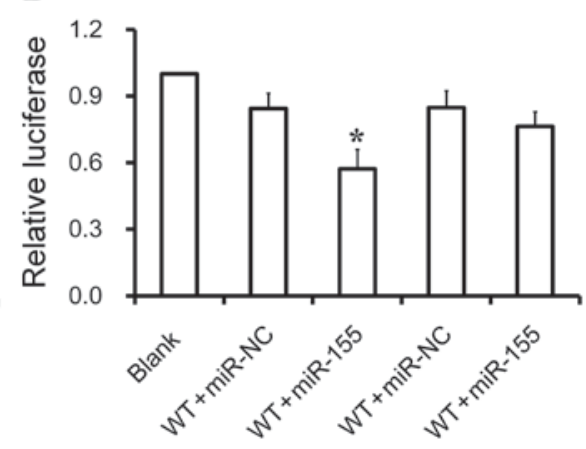

Figure 3. (A) Relative expression level of miR-155 after transfection was assayed by real-time PCR. ${ }^{* *} \mathrm{P}<0.01 \mathrm{vs.} \mathrm{miR} \mathrm{NC.} \mathrm{(B)} \mathrm{Luciferase} \mathrm{reporter} \mathrm{assay} \mathrm{results}$ indicated that SCN1A may be a direct target of miR-155. The luciferase activity of U251 cells transfected with miR-155 mimics was lower in WT SCN1A 3'UTR co-transfected cells compared with cells co-transfected with the MT SCN1A 3'UTR. "P<0.05 vs. WT + miR-NC. miR, microRNA; MT, mutant-type; NC, negative control; SCN1A, sodium voltage-gated channel $\alpha$ subunit 1; UTR, untranslated region; WT, wild-type.
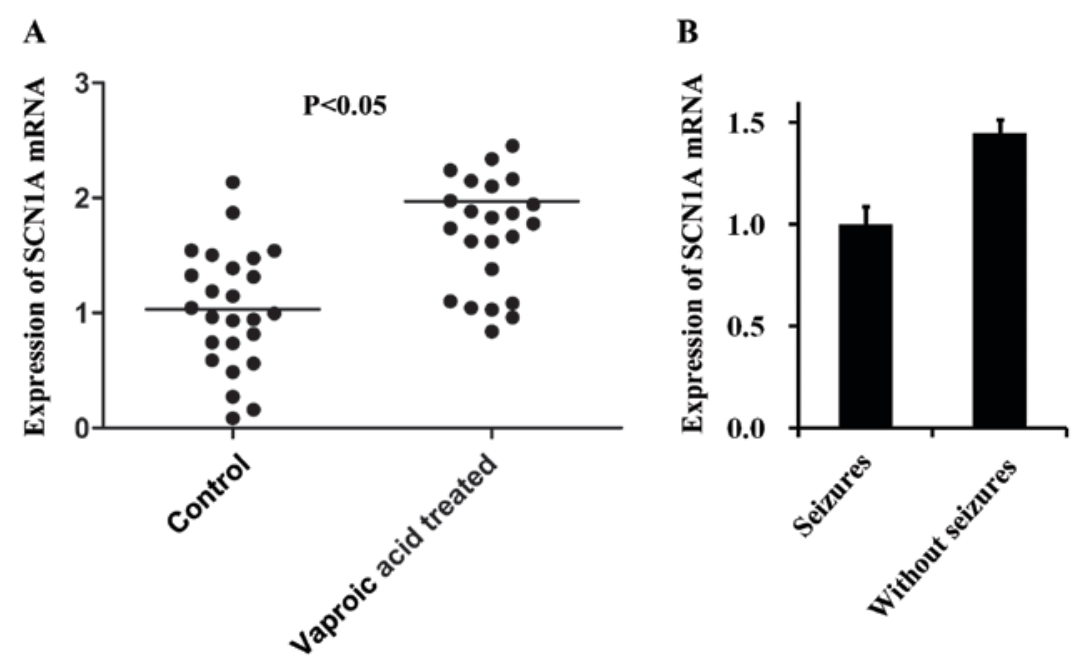

Figure 4. Expression of SCN1A was assayed. (A) SCN1A mRNA expression levels in VPA-treated and control patients. The levels of SCN1A mRNA expression were significantly upregulated in the VPA-treated patients compared with patients in the untreated control group. (B) SCN1A exhibits no difference between patients who experienced seizures and patients who did not experience seizures. SCN1A, sodium voltage-gated channel $\alpha$ subunit 1; VPA, valproic acid.

was lower compared with cells transfected with wild-type SCN1A 3'UTR and miR-NC (Fig. 3B). By contrast, the luciferase activity of cells carrying the mutant SCN1A 3'UTR was comparable with the scramble control and the blank control. These data indicated that SCN1A may be a target of miR-155 in U251 cells with the binding sites located on the SCN1A 3'UTR.

Expression level of SCN1A mRNA varies in VPA-treated and control groups. The SCN1A mRNA expression level was determined in the two treatment groups using RT-qPCR (Fig. 4A). The expression of SCN1A mRNA in the VPA-treated group was higher $(r=0.226 ; P=0.032)$ compared with the expression level in the control group. SCN1A exhibits no difference between patients who experienced seizures and patients who did not experience seizures (Fig. 4B).

Varying expression levels of SCN1A mRNA and protein in different in vitro treatment groups. RT-qPCR and western blot analyses were used to determine the expression of SCN1A mRNA and protein in U251 cells exposed to miR-155 mimics, miR-155 inhibitors or scramble controls. The SCN1A protein (Fig. 5A) and mRNA (Fig. 5B) expression levels were reduced in U251 cells transfected with 50 or $100 \mathrm{nM}$ miR-155 mimics. The results of RT-qPCR showed the same trends. By contrast, cells treated with 50 or $100 \mathrm{nM}$ miR-155 inhibitor exhibited an elevated expression levels of SCN1A protein (Fig. 5C) and mRNA (Fig. 5D) compared with cells treated with the scrambled negative control, and there was no difference between the cells treated with 50 and $100 \mathrm{nM}$ miR-155 inhibitor. These results support a negative regulatory relationship between miR-155 and SCN1A, and suggested a concentration-dependent effect of miR-155 on the expression of SCN1A.

\section{Discussion}

A seizure is an abnormal discharge of neurons in the brain that may follow brain injury that causes transient central nervous system disorders, with sudden and recurrent characteristics that are related to the degree of the brain injury (21). Seizure incidence rate following a severe head injury is $14.8-33.0 \%$; 
A

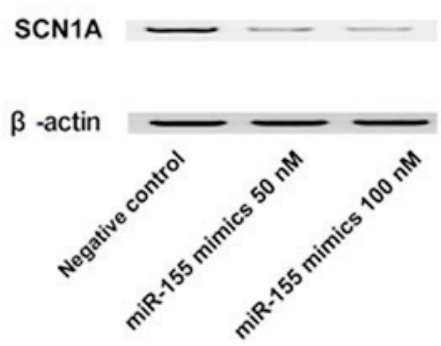

C

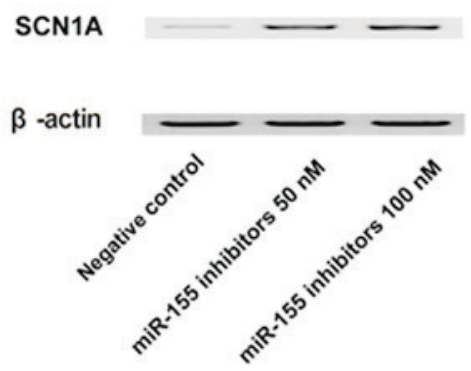

B

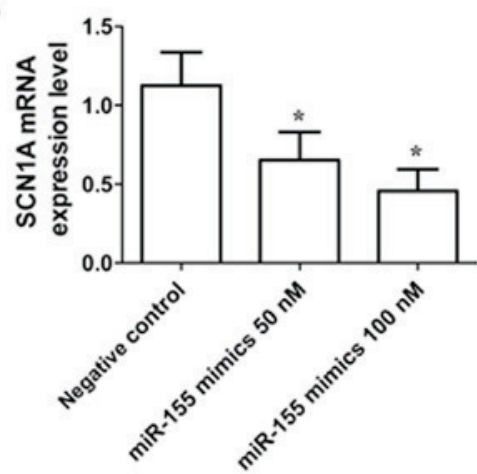

D

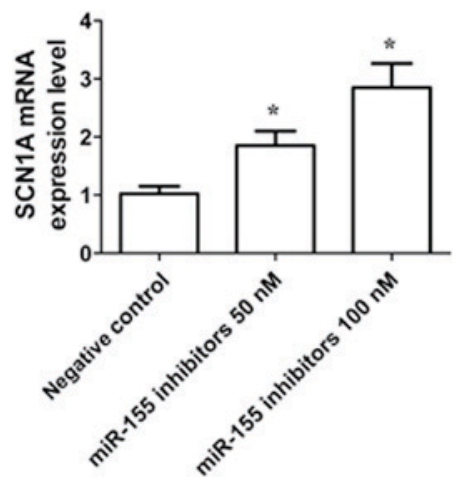

Figure 5. SCN1A protein and mRNA expression analysis in U251 cells subjected to different treatments. SCN1A (A) protein and (B) mRNA expression levels of U251 cells transfected with 50 and $100 \mathrm{nM}$ miR-155 mimics were downregulated compared with the scramble negative control. SCN1A (C) protein and (D) mRNA expression levels of U251 cells transfected with miR-155 inhibitors were markedly elevated compared with the scramble negative controls. ${ }^{*} \mathrm{P}<0.05$ vs. negative control. miR, microRNA; SCN1A, sodium voltage-gated channel $\alpha$ subunit 1 .

patients who need craniotomy and decompressive craniotomy often have cerebral hernia; therefore, their incidence of seizure will be higher $(1,2)$. Clinically, many nerve trauma doctors may not be able to easily diagnose a patient with epilepsy owing to various patient-centric considerations (including schooling, employment, marriage, driving and applying for loans, amongst others); in these situations they will diagnose the patient as suffering from seizures. Furthermore, whether the prophylactic use of AEDs is necessary following neurosurgical surgery has been controversial $(22,23)$.

In the present study, 14 cases of seizures following cranioplasty were observed across the experimental and control groups, with an incidence of $17.5 \%$. Possible associated clinical factors include: i) The cranial cavity volume changes following cranioplasty, resulting in changes to the brain tissue and cerebrospinal fluid dynamics and breaking the relative equilibrium that has existed for several months prior to surgery; ii) some patients may have presented with collapsed bone window tissues prior to surgery, as subdural stitches are often suspended on titanium during surgery to reduce the epidural space, which may cause the arachnoid fibrous band on the brain surface to move the brain tissue and induce changes to brain tissue function; iii) cranioplasty changes the oppression of atmospheric pressure on the brain tissue, bringing alterations to hemodynamics or the external environment of the brain tissue; iv) when patients underwent isolation of the endocranium, 15 cases had damage to the endocranium, of which 12 cases exhibited arachnoid damage and cerebrospinal fluid outflow, although they received a tight suture of the endocranium, non-visible brain damage cannot be excluded; v) the use of some nerve stimulant drugs following surgery, including xingnaojing and naloxone, may also induce seizures $(24,25)$. The incidence rate of cranioplasty-related seizures is relatively high and should be prevented by the use of AEDs (26). In the present study, only 3/41 cases among the experimental group experienced seizures. One of these cases occurred prior to surgery, and was later indicated to be caused by an insufficient plasma concentration of valproic acid; following an increase in dosage, no more seizures were recorded. In two cases, following surgery, the plasma concentration of valproic acid was within expected levels, but could not control seizures. Therefore, the treatment was changed to oxcarbazepine or levetiracetam tablets for seizure control. In the control group, 11/39 patients experienced seizures; all of these were controlled well with VPA. In the experimental and control groups there were no cases that required surgery as a result of a failure to control the seizures. The difference in the incidence rates of seizures between VPA-treated group (7.3\%) and control group (28.2\%) was statically significant $(\mathrm{P}<0.05)$, indicating that VPA may have a preventive effect for cranioplasty-related seizures.

SCN1A is important for the initiation of action potentials in the central nervous system (27). This subunit contains 4 homologous domains (D1-D4) and each contains six transmembrane segments (S1-S6) (28).

The present study revealed that the expression of miR-155 was lower in VPA-treated patients, compared with patients in the untreated control group. Furthermore, computational analysis revealed that SCN1A was a potential target gene of 
miR-155, as it contains a potential seed region in the 3'UTR of the gene, and this was experimentally supported through the use of a luciferase assay. Furthermore, the expression of SCN1A mRNA and protein were estimated in different cell culture treatment groups, which demonstrated that SCN1A protein and mRNA expression levels were reduced in U251 cells in a dose-dependent manner, whereas cells exposed to a miR-155 inhibitor exhibited increased expression levels of SCN1A protein and mRNA. miR-155 has been reported to be involved with several diseases, including seizures. Previous study showed that upregulation of miR-155 was closely associated with ischemic stroke, intracerebral hemorrhage, and kainate seizures (29). Moreover, the expressions of miR-155 were significantly upregulated in the seizure-related acute and chronic stages of mesial temporal lobe epilepsy (MTLE) in the immature rat model and also in children with MTLE (30). Antagonist targeting microRNA-155 protects against lithium-pilocarpine-induced Status Epilepticus in C57BL/6 Mice by activating brain-derived neurotrophic factor (31). And a report also showed that VPA downregulated the expression of miR-155 which had potential protection against cerebral ischemia (6), indicating inhibition of miR-155 is a potential target for seizure and VPA can effectively inhibit the epression of miR-155.

In summary, the incidence of cranioplasty-related seizures is high and is associated with patients' morbidity. Preventative use of VPA in the early postsurgical stages may reduce the incidence of seizures.

\section{References}

1. Lee L, Ker J, Quah BL, Chou N, Choy D and Yeo TT: A retrospective analysis and review of an institution's experience with the complications of cranioplasty. Br J Neurosurg 27: 629-635, 2013.

2. Pechmann A, Anastasopoulos C, Korinthenberg R, van Velthoven-Wurster V and Kirschner J: Decompressive craniectomy after severe traumatic brain injury in children: Complications and outcome. Neuropediatrics 46: 5-12, 2015.

3. Ronen GM, Buckley D, Penney S and Streiner DL: Long-term prognosis in children with neonatal seizures: A population-based study. Neurology 69: 1816-1822, 2007.

4. Mizrahi EM: Neonatal seizures: Problems in diagnosis and classification. Epilepsia 28 (Suppl 1): S46-S55, 1987.

5. Sankar R and Painter MJ: Neonatal seizures: After all these years we still love what doesn't work. Neurology 64: 776-777, 2005.

6. Hunsberger JG, Fessler EB, Wang Z, Elkahloun AG and Chuang DM: Post-insult valproic acid-regulated microRNAs: Potential targets for cerebral ischemia. Am J Transl Res 4: 316-332, 2012

7. Filip A: MiRNA-new mechanisms of gene expression control. Postepy Biochem 53: 413-419, 2007 (In Polish).

8. Chen $\mathrm{K}$ and Rajewsky N: The evolution of gene regulation by transcription factors and microRNAs. Nat Rev Genet 8: 93-103, 2007.

9. Esquela-Kerscher A and Slack FJ: Oncomirs-microRNAs with a role in cancer. Nat Rev Cancer 6: 259-269, 2006.

10. Guarnieri DJ and DiLeone RJ: MicroRNAs: A new class of gene regulators. Ann Med 40: 197-208, 2008.

11. Gauthier BR and Wollheim CB: MicroRNAs: 'Ribo-regulators' of glucose homeostasis. Nat Med 12: 36-38, 2006.
12. Bi Y, Liu G and Yang R: MicroRNAs: Novel regulators during the immune response. J Cell Physiol 218: 467-472, 2009.

13. Hatfield $\mathrm{S}$ and Ruohola-Baker $\mathrm{H}$ : microRNA and stem cell function. Cell Tissue Res 331: 57-66, 2008.

14. Matsubara H, Takeuchi T, Nishikawa E, Yanagisawa K, Hayashita Y, Ebi H, Yamada H, Suzuki M, Nagino $M$, Nimura Y, et al: Apoptosis induction by antisense oligonucleotides against miR-17-5p and miR-20a in lung cancers overexpressing miR-17-92. Oncogene 26: 6099-6105, 2007.

15. Beveridge NJ, Tooney PA, Carroll AP, Gardiner E, Bowden N, Scott RJ, Tran N, Dedova I and Cairns MJ: Dysregulation of miRNA $181 \mathrm{~b}$ in the temporal cortex in schizophrenia. Hum Mol Genet 17: 1156-1168, 2008.

16. Kuhn DE, Nuovo GJ, Martin MM, Malana GE, Pleister AP, Jiang J, Schmittgen TD, Terry AV Jr, Gardiner K, Head E, et al: Human chromosome 21-derived miRNAs are overexpressed in down syndrome brains and hearts. Biochem Biophys Res Commun 370: 473-477, 2008

17. Nicoloso MS and Calin GA: MicroRNA involvement in brain tumors: From bench to bedside. Brain Pathol 18: 122-129, 2008.

18. Zhou R, Yuan P, Wang Y, Hunsberger JG, Elkahloun A, Wei Y, Damschroder-Williams P, Du J, Chen G and Manji HK: Evidence for selective microRNAs and their effectors as common long-term targets for the actions of mood stabilizers. Neuropsychopharmacology 34: 1395-1405, 2009.

19. Goh WW, Oikawa H, Sng JC, Sergot M and Wong L: The role of miRNAs in complex formation and control. Bioinformatics 28: 453-456, 2012

20. Wang L, Wu G, Qin X, Ma Q, Zhou Y, Liu S and Tan Y: Expression of Nodal on bronchial epithelial cells influenced by lung microbes through DNA methylation modulates the differentiation of T-Helper cells. Cell Physiol Biochem 37: 2012-2022, 2015

21. Meyer FB: Calcium, neuronal hyperexcitability and ischemic injury. Brain Res Brain Res Rev 14: 227-243, 1989.

22. Cranley MR, Craner M and McGilloway E: Antiepileptic prophylaxis following severe traumatic brain injury within a military cohort. J R Army Med Corps 162: 109-114, 2016.

23. Klimek M and Dammers R: Antiepileptic drug therapy in the perioperative course of neurosurgical patients. Curr Opin Anaesthesiol 23: 564-567, 2010.

24. Creutzfeldt CJ, Tirschwell DL, Kim LJ, Schubert GB, Longstreth WT Jr and Becker KJ: Seizures after decompressive hemicraniectomy for ischaemic stroke. J Neurol Neurosurg Psychiatry 85: 721-725, 2014.

25. Krause-Titz UR, Warneke N, Freitag-Wolf S, Barth H and Mehdorn HM: Factors influencing the outcome (GOS) in reconstructive cranioplasty. Neurosurg Rev 39: 133-139, 2016.

26. Hesdorffer DC, Benn EK, Cascino GD and Hauser WA: Is a first acute symptomatic seizure epilepsy? Mortality and risk for recurrent seizure. Epilepsia 50: 1102-1108, 2009.

27. Martina M, Vida I and Jonas P: Distal initiation and active propagation of action potentials in interneuron dendrites. Science 287: 295-300, 2000

28. Escayg A, MacDonald BT, Meisler MH, Baulac S, Huberfeld G, An-Gourfinkel I, Brice A, LeGuern E, Moulard B, Chaigne D, et al: Mutations of SCN1A, encoding a neuronal sodium channel, in two families with GEFS+2. Nat Genet 24: 343-345, 2000

29. Liu DZ, Tian Y, Ander BP, Xu H, Stamova BS, Zhan X, Turner RJ, Jickling $G$ and Sharp FR: Brain and blood microRNA expression profiling of ischemic stroke, intracerebral hemorrhage, and kainate seizures. J Cereb Blood Flow Metab 30: 92-101, 2010.

30. Ashhab MU, Omran A, Kong H, Gan N, He F, Peng J and Yin F: Expressions of tumor necrosis factor alpha and microRNA-155 in immature rat model of status epilepticus and children with mesial temporal lobe epilepsy. J Mol Neurosci 51: 950-958, 2013.

31. Cai Z, Li S, Li S, Song F, Zhang Z, Qi G, Li T, Qiu J, Wan J, Sui H and Guo H: Antagonist targeting microRNA-155 protects against lithium-pilocarpine-induced status epilepticus in C57BL/6 mice by activating brain-derived neurotrophic factor. Front Pharmacol 7: 129, 2016 